\title{
Changes in Oxidative Phosphorylation Activity in Fibroblasts at p38 MAPK Pathway Inhibition
}

\author{
Irina A. Shurygina, $\mathrm{PhD}, \mathrm{ScD}^{1^{*}}$; Irina $\mathrm{S}$. Trukhan, $\mathrm{PhD}^{1}$; Nataliya N. Dremina, $\mathrm{PhD}^{1}$; \\ Michael G. Shurygin, $\mathrm{PhD}, \mathrm{ScD}^{2}$ \\ ${ }^{1}$ Irkutsk Scientific Center of Surgery and Traumatology, ${ }^{2}$ Pharmasyntez \\ Irkutsk, the Russian Federation
}

\begin{abstract}
Background: Mitochondrial oxidative phosphorylation (OxPhos) accounts for more than $90 \%$ of the cellular ATP production, plays the role in reactive oxygen species (ROS) generation and programmed cell death. In addition, it contributes to such cellular processes as proliferation, differentiation and cell aging. Currently, several signaling systems are known to participate in regulation of OxPhos and activity of cytochrome c-oxidase $(\mathrm{CcO})$, the terminal enzyme of the mitochondrial electron transport chain. However, data on mechanisms and key units involved in the signal transduction are still being supplemented.

Methods: Peritoneal fibroblasts were isolated from the omentum of Wistar rats by fragmenting the dissected tissue and disaggregating the fragments in collagenase solution $(200 \mathrm{U} / \mathrm{ml})$. The primary culture of fibroblasts was cultured in Dulbecco's Modified Eagle's Medium (DMEM) containing 10\% Fetal Bovine Serum (FBS), $1 \%$ antibiotic/antimycotic at $37^{\circ} \mathrm{C}, 80 \% \mathrm{RH}$ and $5 \% \mathrm{CO}_{2}$ in Biostation CT, Nikon. To obtain a culture, the fibroblasts were subcultured every 7 days. After the third passage the culture was treated with SB203580 at the concentration of $10 \mu \mathrm{M}$ or with SB203580 in combination with bFGF at the dose of 133 $\mathrm{pg} / \mathrm{ml}$. Cells for immunofluorescent studies were fixed with 70\% ethanol and stained with antibodies to CcO subunit I.

Results: When exposed to SB203580 or the combination of SB203580 and bFGF, marked changes were observed in the fibroblast culture: in both cases there was intensive collagen destruction; attached fibroblasts rounded and detached from the substrate. When exposed to SB203580, non-rounded cells started to vacuolate actively while vacuoles occupied the entire cytoplasm. Introduction of the p38 inhibitor into the culture of activated fibroblasts caused a more intensive cell detachment and collagen destruction. Moreover, the formation of large conglomerates containing several dozens of cells connected with collagen fibers was observed in the areas characterized by the highest cell density. Immunofluorescent staining made it possible to reveal a certain increase in the cell area occupied by CcO after fibroblasts exposure to SB203580 and a significant increase in the area of the enzyme distribution in the studied cells (more than 5-fold in comparison with the control group) when simultaneously adding SB203580 and bFGF. In addition, one-way ANOVA test demonstrated a statistically significant increase in the CcO fluorescent staining intensity in both cases.

Conclusion: The analysis of the results indicates that SB203580, a p38 MAPK inhibitor, influences both peritoneal fibroblast morphology and the energy status of the cells under study increasing the amount of $\mathrm{CcO}$, the terminal enzyme of the mitochondrial electron transport chain, in the cells, although no cell change to active proliferation or apoptosis was observed. Fibroblast culture stimulation by bFGF significantly enhances the effect of SB203580, which implies a greater OxPhos complex expression in case of impaired p38 MAPK signaling pathway activation. (International Journal of Biomedicine. 2019;9(4):350-355.)
\end{abstract}

Key Words: peritoneal fibroblast $\bullet$ oxidative phosphorylation $\bullet$ p38 MAPK $・$ SB203580 • bFGF $\bullet$ OxPhos

\section{Abbreviations}

ATP, adenosine triphosphoric acid; bFGF, basic fibroblast growth factor; cAMP, 3',5'-cyclic adenosine monophosphate; CcO, cytochrome c-oxidase; CHO cell line, Chinese hamster ovary cell line; DMEM, Dulbecco's Modified Eagle's Medium; EGFR, Epidermal Growth Factor Receptor; ERK, extracellular signal-regulated kinase; FBS, Fetal Bovine Serum; IL-1, Interleukin-1; MAPK, mitogen-activated protein kinases; MMP, matrix metalloproteinase; OxPhos, oxidative phosphorylation; ROS, reactive oxygen species, TGF- $\boldsymbol{\beta}$, Transforming growth factor beta, TNF $\boldsymbol{\alpha}$, tumor necrosis factor alpha. 


\section{Introduction}

Under normal physiological conditions mitochondrial OxPhos accounts for more than $90 \%$ of the cellular ATP production in most cells and tissues. Mitochondria are also involved in the maintenance of calcium homeostasis, carry out critical reaction steps of steroid hormone metabolism, pyrimidine synthesis and elimination of ammonia through the urea cycle. Moreover, they are considered one of the major programmed cell death modulators and the source of reactive oxygen species. ${ }^{(1-3)}$

That is why mitochondrial dysfunctions caused by defective electron transport complexes or by dysfunction of separate chain elements are linked to a variety of diseases and pathological conditions of the humans, including so-called mitochondrial diseases (Alzheimer's disease and Parkinson's disease), cancer, diabetes, cardiovascular diseases, as well as myocardial ischemia/reperfusion. ${ }^{(2)}$ Normally, OxPhos regulation also has a great impact on such key processes as cell differentiation and cell aging. So, it was shown that formation of myofibroblasts is closely linked to metabolism switching from OxPhos to glycolysis. ${ }^{(4)}$ Studies conducted on skinderived fibroblasts revealed age-related decline in efficiency of OxPhos. ${ }^{(5)}$ The role of OxPhos in cell proliferation still remains a subject of debate. Along with the data saying that OxPhos in actively proliferating cells, both tumor cells and non-oncogenic actively proliferating cells (non-transformed fibroblasts, lymphocytes, macrophages, thymocytes, endothelial cells and embryonic stem cells ${ }^{(6,7)}$ is inhibited and energy metabolism is carried out by means of glycolysis and pentose phosphate pathway even in the presence of oxygen (the Warburg effect), ${ }^{(2,8)}$ there are studies demonstrating an increase in OxPhos in the cells of certain cancer types (including leukaemias, lymphomas, pancreatic ductal adenocarcinoma, melanoma and endometrial carcinoma), ${ }^{(9)}$ as well as an increase in OxPhos during active proliferation of fibroblasts. ${ }^{(7)}$

Mitochondrial complex IV or $\mathrm{CcO}$ is the terminal enzyme of the electron transport chain. It catalyzes the electron and proton transfer across the membrane to molecular oxygen to make $\mathrm{H}_{2} \mathrm{O}$. CcO is also one of the three $\mathrm{H}^{+}$pumps (along with complexes I and III) that generate the proton gradient across the inner mitochondrial membrane, which powers the ATP synthesis. ${ }^{(2,3)}$ Besides energy function, $\mathrm{CcO}$ also contributes to ROS generation during oxidative stress, although the data about the potential role of this enzyme in the process are contradictory. On the one hand, it is known that $\mathrm{CcO}$ dysfunction or enzyme deficit increases reactive oxygen species generation (for example, an increased ROS production was observed in the cells with enzyme knock-out mRNA). This is explained by the fact that even though $\mathrm{CcO}$ does not participate directly in ROS generation it can affect their generation by consuming the electrons that could have been involved in oxygen activation. ${ }^{(10,11)}$ On the other hand, there was a certain volume of evidence published suggesting that $\mathrm{CcO}$ subunit I can exhibit pro-oxidant properties and the hydrogen peroxide sensitivity of cells increases with overexpression of $\mathrm{CcO}$ subunit $\mathrm{I}^{(12)} \mathrm{In}$ addition, under hypoxic conditions complex IV can participate in NO production thus mediating the impact on NO-dependent signaling pathways. ${ }^{(13)}$

It is known that both under normal and pathological conditions several signaling pathways are involved in OxPhos regulation and $\mathrm{CcO}$ activity. However, the data about the mechanisms and various molecules participating in the signal transduction are still being supplemented. The molecules affecting $\mathrm{CcO}$ activity are the thyroid hormone, 5 -diiodothyronine (T2) that activates the enzyme even in the presence of an allosteric ATP inhibitor; ${ }^{(2,3)}$ glucagon that has a suppressive effect; ${ }^{(14,15)}$ EGFR, which is, when activated, translocated to cytoplasm and localized in the mitochondrial membrane where it phosphorylates $\mathrm{CcO}$ subunit II, which leads to a partial inhibition of its activity and cell transition from the aerobic to glycolytic and pentose phosphate pathways; ${ }^{(16)}$ Smad4, a negative regulation mediator in TGF- $\beta$ signaling that binds with $\mathrm{CcO}$ during apoptosis. ${ }^{(17)} \mathrm{CcO}$ is competitively inhibited by nitrogen oxide (NO), carbon monoxide (CO), noncompetitively inhibited by hydrogen sulphide $\left(\mathrm{H}_{2} \mathrm{~S}\right)$, hydrogen cyanide $(\mathrm{HCN})$ and sodium azide $\left(\mathrm{NaN}_{3}\right)^{(2,3)}$ Mitochondrial complex IV is also a target for cAMP-dependent regulation, in addition, increasing cAMP levels in $\mathrm{CHO}$ cells led to inhibition of $\mathrm{CcO}$ activity under hypoxic conditions and in case of ischemia/reperfusion; ${ }^{(2,14,15)}$ the enzyme activity is also inhibited by $\mathrm{TNF} \alpha$, a pro-inflammatory cytokine, and cytochrome P450. ${ }^{(3,18)}$

The goal of this study was to investigate the impact of the p38 MAPK signaling pathway on OxPhos activity of peritoneal fibroblasts in culture.

\section{Materials and Methods}

Primary fibroblast culture isolation from the rat omentum

The experiment for isolation of primary fibroblast culture from the omentum of mature Wistar rats (200 g) was carried out. Animals were housed in accordance with the Good Laboratory Practice (GLP) rules. The experiments were performed in accordance with the norms for the humane treatment of animals regulated by the International Guidelines of the Association for the Assessment and Accreditation of Laboratory Animal Care in accordance with the protocol approved by the Institutional Animal Care and Use Committee of the Irkutsk Scientific Center of Surgery and Traumatology. All the operative interventions were conducted aseptically. Animals were anesthetized with an intramuscular injection of $2 \%$ rometar at the dose of $0.2 \mathrm{~mL} / \mathrm{kg}$.

The primary culture was obtained by fragmenting the dissected omentum and disaggregating the tissue fragments at $37^{\circ} \mathrm{C}$ in the solution containing $200 \mathrm{U} / \mathrm{ml}$ of collagenase, $2 \%$ antibiotic/antimycotic $(10,000 \mathrm{U} / \mathrm{ml}$ of penicillin, $10,000 \mu \mathrm{g} /$ $\mathrm{ml}$ of streptomycin and $25 \mu \mathrm{g} / \mathrm{ml}$ of amphotericin B, Gibco) in DMEM. Collagenase activity in the suspension was inhibited by equal amount of DMEM containing 15\% FBS (SigmaAldrich) and 1\% antibiotic/antimycotic. Then the cells were twice washed in DMEM spiked with $10 \%$ FBS, $1 \%$ antibiotic/ antimycotic by centrifuging the suspension at $500 \mathrm{G}$ for 5 minutes. Isolated fibroblasts were cultured in DMEM containing $10 \%$ FBS, $1 \%$ antibiotic/antimycotic at $37^{\circ} \mathrm{C}, 80 \% \mathrm{RH}$ and $5 \%$ 
$\mathrm{CO}_{2}$ in the Biostation CT, Nikon. To obtain a culture, fibroblasts were subcultured every 7 days.

After the third passage the culture was treated with SB203580 (4-[5-(4-fluorophenyl)-2-[4-(methylsulfonyl)phenyl]$1 \mathrm{H}$-imidazol-4-yl]pyridine) at the concentration of $10 \mu \mathrm{M}$, bFGF at the dose of $133 \mathrm{pg} / \mathrm{ml}$ or their combination. The cells that were not exposed to the active substances served as controls (an appropriate amount of DMEM was added to the culture).

Immunofluorescence staining

Cells for immunofluorescence studies were fixed with $70 \%$ ethanol and stained with antibodies to $\mathrm{CcO}$ (anti-OxPhos Complex IV subunit I monoclonal antibody, Invitrogen, Cat. ND 0589, Lot 459600) in a dilution of 1:200. Alexa fluor 568 goat anti-mouse $\operatorname{IgG}(\mathrm{H}+\mathrm{L})$ (Invitrogen, Cat. NA-11031, Lot 822389 ) in a dilution of 1:300 was used as secondary antibody. Nuclei were stained with Hoechst (Invitrogen, Cat. NH-3570, Lot822389), 1:300. ${ }^{(19,20)}$

\section{Results}

Morphological changes in peritoneal fibroblast culture

At the first stage of the study, we derived primary fibroblast culture from the rat omentum. After 7 days, the cells were subcultured to obtain a pure culture, and after three passages, the fibroblasts were introduced in the experiment.

To activate the cells, bFGF at the dose of $133 \mathrm{pg} / \mathrm{ml}$ was added. 30 minutes after exposure of fibroblast culture to bFGF, marked changes were observed (intensive collagen destruction, collagen fibers started to grow thinner). At the same time, some rounding of the attached fibroblasts was observed, some of them detached from the matrix and moved freely in the culture medium. A formation of conglomerates containing several dozens of fibroblasts was observed in the areas characterized by the highest cell density (Fig. 1 A, 1B). During the next day, the cell conglomerates thickened, producing more compact formations, which, however, disintegrated upon mechanical action (when moving the culture bottle or adding the medium).

To block the signaling of the p38 MAPK pathway, cells were exposed to SB203580, the mitogen-activated protein kinase inhibitor, at the concentration of $10 \mu \mathrm{M}$. Both the intact culture and the one activated by fibroblast growth factor (133 $\mathrm{pg} / \mathrm{ml}$ ) were subjected to the exposure. 30 minutes later, marked changes were observed in the fibroblast culture: in both cases there was intensive collagen destruction, collagen fibers started to grow thinner; attached fibroblasts rounded, detached from the substrate and moved freely in the culture medium. When exposed to SB203580, 2 hours after the substance adding, non- rounded cells started to vacuolate actively with vacuoles occupying the entire cytoplasm (Figure 1C). Introduction of two active substances into the fibroblast culture caused more intensive cell detachment and collagen destruction. Moreover, the formation of large conglomerates containing several dozens of cells connected with collagen fibers was observed in the areas characterized by the highest cell density (Figure 1D). During the next day, the cell conglomerates thickened, producing more compact formations, which disintegrated upon mechanical action (when moving the culture bottle or adding the medium) and broke up into separate cells or smaller cell clusters.

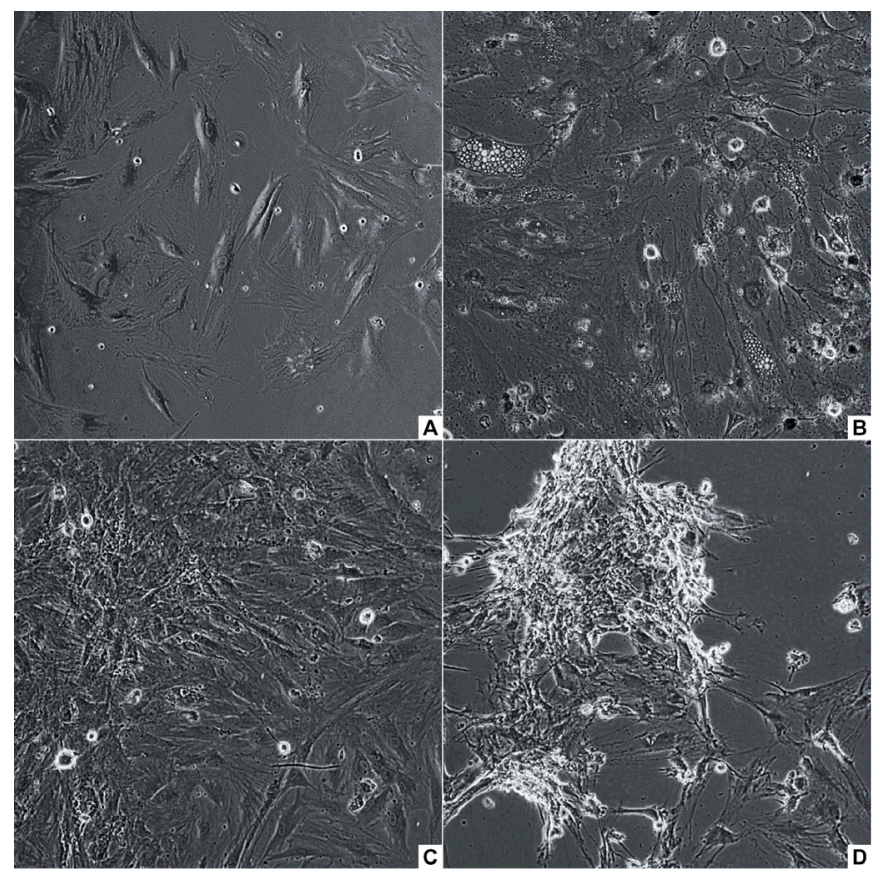

Fig. 1. Fibroblasts with dense intercellular junction and high content of collagen fibers before exposure to bFGF (A); Fibroblast conglomerate 30 minutes after exposure to bFGF(B); Fibroblast conglomerates 30 minutes after exposure to $S B 203580$ (C) and SB203580 in combination with bFGF (D).

Immunofluorescence studies

To study the OxPhos activity, cultured cells were fixed and stained with antibodies to mitochondrial $\mathrm{CcO}$ subunit I on Day 3 after exposure to active substances. Nuclei were stained with Hoechst.

Specific color staining of distinctly structured ovalshaped nuclei was observed in the controls. Mitochondrial complex IV was localized pointwise in the cells in direct proximity to the nuclei (Figure 2A).

After immunofluorescence staining of fibroblasts cultured with bFGF, irregular-shaped nucleus content with different Hoechst staining intensity was visualized. At the same time, the color of the mitochondrial complex IV in fibroblasts was uneven and was absent in most of the cells under study (Figure 2B).

After fibroblast cultivation with p38 MAPK inhibitor SB203580, the color of the mitochondrial complex IV in certain cells became more intense, being localized in the entire cell and represented by numerous pointwise formations. Nucleus content was characterized by irregular shape and indistinct contours and the Hoechst staining intensity was different (Figure 2C).

Fibroblasts exposed to p38 MAPK inhibitor in combination with bFGF were characterized by even more intense $\mathrm{CcO}$ staining. In this case, OxPhos fluorescence occupied a larger part of the cell volume, partially overlapping the color of the nuclei. At the same time, the cell nuclei were characterized by a more pale color and less structured content as well as by indistinct contours (Figure 2D). 

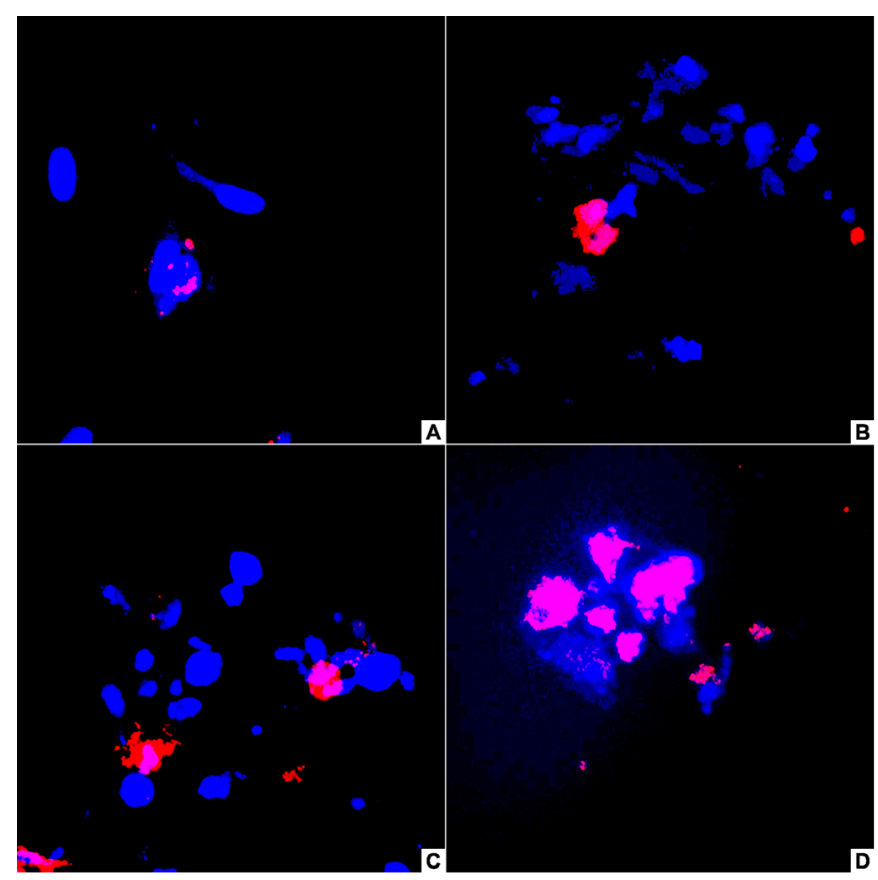

Fig. 2. OxPhos fluorescence staining (red color). Fibroblast, control group (A), Fibroblasts with bFGF (B), SB203580 effect on fibroblasts (C), SB203580 and bFGF effect on fibroblasts (D). Hoechst (blue color).

Statistical analysis of the data obtained also confirmed a significant increase (more than 5-fold) in the area of the cell stained with antibodies to mitochondrial complex IV when fibroblasts were exposed to the combination of SB203580 and bFGF, whereas introducing only the p38 MAPK inhibitor into the culture did not significantly increase the area of the fluorescently stained enzyme in the cultured cells (Figure 3). Uneven staining observed in the cells under study and, accordingly, a wide interquartile range in the analyzed cell groups are associated with heterogeneity of the resulting culture (cultured fibroblasts are at different stages of the cell cycle).

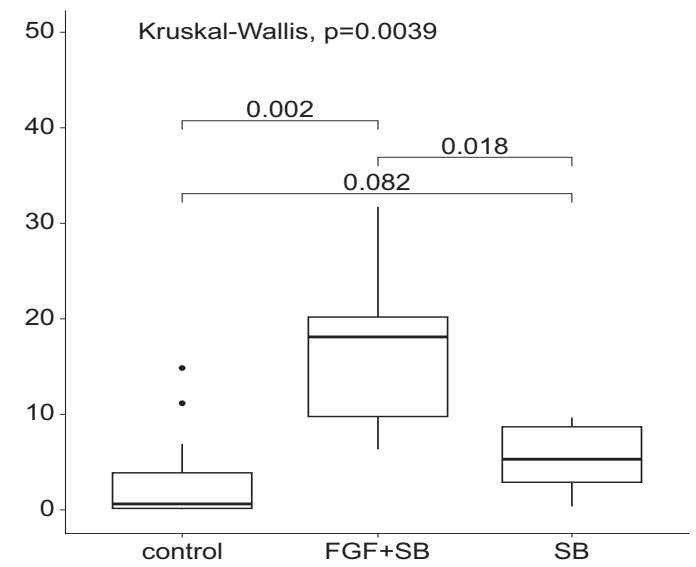

Fig. 3. One-way analysis of variance (Kruskal-Wallis test) of the changes in the area of the cell occupied by $\mathrm{CcO}$ in the controls and the samples treated with SB203580 as well as with the combination of SB203580 and bFGF. A pairwise comparison was made using the Wilcoxon test. Median values, the 1st and the $3 d$ quartiles are presented. Differences are considered statistically significant at $P<0.05$.
At the same time one-way analysis of variance showed that total fluorescence staining intensity of complex IV is significantly greater through exposure to SB203580, both in intact cells and in bFGF-activated fibroblasts: 6 and 4 times more, respectively. This may suggest that the concentration of $\mathrm{CcO}$ in mitochondria increases in p38 MAPK inhibitorincubated fibroblasts. However, the intracellular distribution of these organelles in the cell is quite tight, whereas after incubation of bFGF-activated fibroblasts with SB203580, there is less increase in the concentration of the OxPhos complex in mitochondria but its intracellular localization becomes far more distributed.

\section{Discussion}

MAPKs constitute a protein family relaying signal transduction, amplification and integration and thus providing for appropriate physiological responses of mammalian cells, such as proliferation, differentiation, inflammation and apoptosis. ${ }^{(21-24)}$ One of the MAP kinase subfamilies, p38 MAPK, is known to be activated under stress conditions caused by ultraviolet radiation, heat shock, osmotic stress, lipopolysaccharide, protein synthesis inhibitors, pro-inflammatory cytokines (IL-1, TNF- $\alpha$, etc.), and also by certain mitogens realizing the effect through tyrosine kinase receptors in particular. In response to the stimulus, p38 MAPK system can interrupt the cell cycle in the G1/S phase during mitotic spindle formation, thus launching one of the physiological response scenarios. ${ }^{(25)}$ For example, p38 MAPK is known to be involved in the differentiation of adipocytes, cardiomyocytes, chondroblasts, erythroblasts, myoblasts and neurons. ${ }^{(26)}$

The association of p38 MAPK with OxPhos level and $\mathrm{CcO}$ activity in particular, is underexplored, however, there is some evidence that genetic knockout of p38 in Purkinje neurons suppressed the mitochondrial respiration in male mice while increasing $\mathrm{CcO}$ expression in female mice. ${ }^{(27)}$ Ronda et al. (2010) report on p38 MAPK-mediated effects of estradiol on the muscle cells treated with hydrogen peroxide indicating that MAPK inhibition leads to inability of the hormone to prevent cell damage exerted by oxidative stress, while mitochondrial membrane disintegration was determined by measuring $\mathrm{CcO}$ activity in the cytosol. ${ }^{(28)}$ In addition, the study conducted on hepatocarcinoma cells demonstrated a positive dependence of $\mathrm{CcO}$ subunit IV expression from $\mathrm{p} 38$ MAPK activity. ${ }^{(29)}$

Our results demonstrate an increase in $\mathrm{CcO}$ levels during p38 MAPK inhibition, which may indicate that p38 MAPK negatively regulates the $\mathrm{CcO}$ expression in peritoneal fibroblasts of adult animals (Figure 2C).

bFGF performs numerous functions in the body, it participates in wound repair, stimulates angiogenesis both under normal and pathological conditions (carcinogenesis), influences vascular tone and blood pressure, participates in inflammatory responses, is essential to normal fetal development (since it induces embryonic and extra-germ cell division and is of great importance for limbs development), plays a role in the brain cortex development as well. In addition, FGF is one of the cell proliferation and cell death suppression 
mediators during carcinogenesis. ${ }^{(30,31)}$ At the cellular level, FGF stimulates fibroblast and endothelial cells proliferation, promotes migration of endotheliocytes regulating proteolysis and adhesion molecule expression. ${ }^{(31,32)}$

FGF and 338 MAPK interaction has not been well-defined, but there is some evidence of these molecules interaction in the regulation of the processes associated with cell proliferation and differentiation. Thus, Matsumoto et al. ${ }^{(33)}$ investigated the role of p38 activation in FGF-2-stimulated angiogenesis: in collagen gel cultures, bovine capillary endothelial cells formed tubular growth-arrested structures in response to FGF-2, while FGF induced more potent MAPK activation. Treatment with the p38 inhibitor, however, enhanced cell morphogenesis and increased cell proliferation. ${ }^{(33)}$ There is also data indicating that fibroblast growth factor (at the concentration of $100 \mathrm{ng} / \mathrm{ml}$ ) affects chondrocyte proliferation and differentiation inhibiting cell multiplication both in vitro and in vivo, whereas regulation was mediated by ERK1/2 and p38 MAPK signaling pathways. In this case, the use of MAPK inhibitors prevented FGF-induced growth of chondrocytes. ${ }^{(34)}$ In addition, p38 MAPK is essential to FGF-induced proliferation of fibroblast-like 3T3 mouse cells. (35) At the same time, the sustained p38 MAPK activation is crucial to FGF-induced cell death in cells of Ewing's sarcoma as compared to transient $\mathrm{p} 38 \mathrm{MAPK}$ activation, which mediates FGF-induced cell proliferation. ${ }^{(36)}$

In the study under discussion, SB203580, a p38 MAPK inhibitor, enhanced the effect of the fibroblast growth factor by increasing the area occupied by $\mathrm{CcO}$ in the cultured fibroblasts.

In addition to participating in the transduction of signals that cause cell proliferation and differentiation, p38 MAPK is also a modulator of metalloproteinase synthesis regulation, which provide for remodeling of collagen extracellular matrix. For example, synthesis and secretion of collagenase-1 (MMP1) expressed by several cell types including fibroblasts, MMP-3, expressed in lamellar epithelial cells and MMP-9 of sarcoma cells depend on the p38 MAPK activity. ${ }^{(37)}$

In our paper, p38 MAPK inhibition in combination with fibroblast growth factor introduction could have caused impaired elimination of extracellular collagen, which resulted in incomplete collagen utilization by enzymes during cell rounding and detachment and the remaining fibrils contributed to cell conglomerate formation.

Thus, the analysis result indicates that p38 MAPK activity inhibition influences both fibroblast morphology and the energy status of the cells under study increasing the amount of $\mathrm{CcO}$, the terminal enzyme of the mitochondrial electron transport chain, in the cells, although no cell change to active proliferation or apoptosis was observed.

\section{Competing Interests}

The authors declare that they have no competing interests.

\section{References}

1. Jezek P, Hlavata L. Mitochondria in homeostasis of reactive oxygen species in cell, tissues, and organism. Int $\mathrm{J}$ Biochem. Cell Biol. 2005;37(12):2478-503.
2. Hüttemann M, Lee I, Grossman LI, Doan JW, Sanderson TH. Phosphorylation of mammalian cytochrome $\mathrm{C}$ and cytochrome $\mathrm{C}$ oxidase in the regulation of cell destiny: respiration, apoptosis, and human disease. Adv Exp Med Biol. 2012;748:237-64. doi: 10.1007/978-1-4614-3573-0 10.

3. Srinivasan S, Avadhani NG. Cytochrome c oxidase dysfunction in oxidative stress. Free Radic Biol Med. 2012;53(6):1252-63.doi:10.1016/j.freeradbiomed.2012.07.021. 4. Vallée A, Lecarpentier Y, Vallée JN. Thermodynamic aspects and reprogramming cellular energy metabolism during the fibrosis process. Int J Mol Sci. 2017;18(12). pii: E2537. doi: 10.3390/ijms18122537

5. Greco M, Villani G, Mazzucchelli F, Bresolin N, Papa $\mathrm{S}$, Attardi G. Marked aging-related decline in efficiency of oxidative phosphorylation in human skin fibroblasts. FASEB J. 2003;17(12):1706-8. doi: 10.1096/fj.02-1009fje.

6. Vander Heiden MG, Cantley LC, Thompson CB. Understanding the Warburg effect: the metabolic requirements of cell proliferation. Science. 2009;324(5930):1029-33. doi: 10.1126/science.1160809.

7. Yao CH, Wang R, Wang Y, Kung CP, Weber JD, Patti GJ. Mitochondrial fusion supports increased oxidative phosphorylation during cell proliferation. Elife. 2019;8. pii: e41351. doi: 10.7554/eLife.41351.

8. Abdel-Haleem AM, Lewis NE, Jamshidi N, Mineta K, Gao X, Gojobori T. The emerging facets of non-cancerous warburg effect. Front Endocrinol (Lausanne). 2017;8:279. doi: 10.3389/fendo.2017.00279.

9. Ashton TM, McKenna WG, Kunz-Schughart LA, Higgins GS. Oxidative phosphorylation as an emerging target in cancer therapy. Clin Cancer Res. 2018;24(11):2482-90. doi: 10.1158/1078-0432.CCR-17-3070.

10. Dawson TL, Gores GJ, Nieminen AL, Herman B, Lemasters JJ. Mitochondria as a source of reactive oxygen species during reductive stress in rat hepatocytes. Am J Physiol. 1993; 264(4 Pt 1):C961-7. doi: 10.1152/ajpcell.1993.264.4.C961.

11. Fukuda R, Zhang H, Kim JW, Shimoda L, Dang CV, Semenza GL. HIF-1 regulates cytochrome oxidase subunits to optimize efficiency of respiration in hypoxic cells. Cell. 2007;129(1):111-22. doi: 10.1016/j.cell.2007.01.047.

12. Khalimonchuk O, Bird A, Winge DR. Evidence for a prooxidant intermediate in the assembly of cytochrome oxidase. J Biol Chem. 2007;282(24):17442-9. doi: 10.1074/jbc.M702379200.

13. Castello PR, David PS, McClure T, Crook Z, Poyton RO. Mitochondrial cytochrome oxidase produces nitric oxide under hypoxic conditions: implications for oxygen sensing and hypoxic signaling in eukaryotes. Cell Metab. 2006;3:27787. doi: 10.1016/j.cmet.2006.02.011.

14. Yang WL, Iacono L, Tang WM, Chin KV. Novel function of the regulatory subunit of protein kinase A: regulation of cytochrome c oxidase activity and cytochrome c release. Biochemistry. 1998; 37(40):14175-80. doi: 10.1021/bi981402a. 15. Lee I, Salomon AR, Ficarro S, Mathes I, Lottspeich F, Grossman LI, Huttemann M. cAMP-dependent tyrosine phosphorylation of subunit I inhibits cytochrome c oxidase activity. J Biol Chem. 2005; 280(7):6094-100. doi: 10.1074/ jbc.M411335200.

16. Boerner JL, Demory ML, Silva C, Parsons SJ.

*Corresponding author: Prof. Irina A. Shurygina, PhD, ScD. Irkutsk Scientific Center of Surgery and Traumatology. Irkutsk, the Russian Federation, E-mail: irinashurygina@gmail.com 
Phosphorylation of Y845 on the epidermal growth factor receptor mediates binding to the mitochondrial protein cytochrome $\mathrm{c}$ oxidase subunit II. Mol Cell Biol. 2004;24(16):7059-71. doi: 10.1128/MCB.24.16.7059-7071.2004.

17. Pang L, Qiu T, Cao X, Wan M. Apoptotic role of TGF- $\beta$ mediated by Smad4 mitochondria translocation and cytochrome c oxidase subunit II interaction. Exp Cell Res. 2011;317(11):1608-20. doi: 10.1016/j.yexcr.2011.02.004.

18. Samavati L, Lee I, Mathes I, Lottspeich F, Huttemann M. Tumor necrosis factor alpha inhibits oxidative phosphorylation through tyrosine phosphorylation at subunit I of cytochrome c oxidase. J Biol Chem. 2008;283(30):21134-44. doi: 10.1074/ jbc.M801954200.

19. Shurygin MG, Shurygina IA, Kanya OV, Dremina NN, Lushnikova EL, Nepomnyashchikh RD. Morphological evaluation of oxidative phosphorylation system in myocardial infarction under conditions of modified vascular endothelial growth factor concentration. Bull Exp Biol Med. 2015;159 (3):402-5. doi: 10.1007/s10517-015-2974-X.

20. Shurygin MG, Shurygina IA, Granina GB, Zelenin NV, Ayushinova NI. Using laser confocal microscopy to assess the activity of MAP kinase systems in the reparative process. Bulletin of the Russian Academy of Sciences: Physics. 2016;.80(1):14-6. doi: 10.3103/S1062873816010214.

21. Zhang W, Liu HT. MAPK signal pathways in the regulation of cell proliferation in mammalian cells. Cell Res. 2002;12(1):9-18. doi: 10.1038/sj.cr.7290105.

22. Shurygina IA, Shurygin MG, Ayushinova NI, Granina GB, Zelenin NV. Mechanisms of connective tissue formation and blocks of mitogen activated protein kinase. Frontiers of Chemical Science and Engineering. 2012;6(2):232-7. doi: 10.1007/s11705-012-1286-1.

23. Shurygina IA, Shurygin MG, Zelenin NV, Ayushinova NI Influence on mitogen-activated protein kinases as a new direction of connective tissue growth regulation. Byulleten Sibirskoy Meditsiny. 2017;16(4):86-93. doi: 10.20538/16820363-2017-4-86-93.

24. Shurygina IA, Aushinova NI, Shurygin MG. Effect of p38 MAPK inhibition on apoptosis marker expression in the process of peritoneal adhesion formation. International Journal of Biomedicine. 2018;8(4):342-6. doi: 10.21103/ Article8(4)_OA15.

25. Takenaka K, Mcriguchi T, Nishida E. Activation of the protein kinase p38 in the spindle assembly checkpoint and mitotic arrest. Science. 1998;280(5363):599-602. doi: 10.1126/science. 280.5363 .599 .

26. Molkentin JD, Bugg D, Ghearing N, Dorn LE, Kim P, Sargent MA et al. Fibroblast-specific genetic manipulation of p38 mitogen-activated protein kinase in vivo reveals its central regulatory role in fibrosis. Circulation. 2017;136(6):549-61. doi: 10.1161/CIRCULATIONAHA.116.026238.

27. Ju X, Wen Y, Metzger D, Jung M. The role of p38 in mitochondrial respiration in male and female mice. Neurosci Lett. 2013;544:152-6. doi: 10.1016/j.neulet.2013.04.004.

28. Ronda AC, Vasconsuelo A, Boland R. Extracellularregulated kinase and p38 mitogen-activated protein kinases are involved in the antiapoptotic action of 17beta-estradiol in skeletal muscle cells. J Endocrinol. 2010;206(2):235-46. doi: 10.1677/JOE-09-0429.

29. Lu Z, Zhou H, Zhang S, Dai W, Zhang Y, Hong L et al. Activation of reactive oxygen species-mediated mitogenactivated protein kinases pathway regulates both extrinsic and intrinsic apoptosis induced by arctigenin in Hep G2. J Pharm Pharmacol. 2020;72(1):29-43. doi: 10.1111/jphp.13180.

30. Powers CJ, McLeskey SW, Wellstein A. Fibroblast growth factors, their receptors and signaling. Endocr Relat Cancer. 2000;7(3):165-97.

31. Shurygin MG, Shurygina IA. Influence of FGF2 level on the inflammation phase dynamics at the postinfarction cardiosclerosis. Patol Fiziol Eksp Ter. 2010;(4):34-7.

32. Shurygin MG, Kanya OV, Dremina NN, Shurygina IA. Morphometric analysis of the growth factors influence on fibroblastic phase of inflammation at experimental myocardial infraction. Acta Biomedica Scientifica. 2014.97(3):105-8.

33. Matsumoto T, Turesson I, Book M, Gerwins P, ClaessonWelsh L. p38 MAP kinase negatively regulates endothelial cell survival, proliferation, and differentiation in FGF-2stimulated angiogenesis. J Cell Biol. 2002;156(1):149-60. doi: 10.1083/jcb.200103096.

34. Raucci A, Laplantine E, Mansukhani A, Basilico C. Activation of the ERK1/2 and p38 mitogen-activated protein kinase pathways mediates fibroblast growth factor-induced growth arrest of chondrocytes. J Biol Chem. 2004;279(3):174756. doi: 10.1074/jbc.M310384200.

35. Maher P. p38 mitogen-activated protein kinase activation is required for fibroblast growth factor-2-stimulated cell proliferation but not differentiation. J Biol Chem. 1999;274(25):17491-8. doi: 10.1074/jbc.274.25.17491.

36. Williamson AJ, Dibling BC, Boyne JR, Selby P, Burchill SA. Basic fibroblast growth factor-induced cell death is effected through sustained activation of p38MAPK and upregulation of the death receptor p75NTR. J Biol Chem. 2004;279(46):47912-28. doi: 10.1074/jbc.M409035200.

37. Johansson N, Ala-aho R, Uitto V, Grénman R, Fusenig NE, López-Otín C, Kähäri VM. Expression of collagenase-3 (MMP-13) and collagenase-1 (MMP-1) by transformed keratinocytes is dependent on the activity of p38 mitogenactivated protein kinase. J Cell Sci. 2000;113 Pt 2:227-35. 